\title{
Product development of sugar palm composites: from concept to fabrication
}

\begin{abstract}
Chapter 11 presents the development process relating to the conceptual design of sugar palm fibre-reinforced polymer matrix composites. The conceptual design is performed by employing several design tools, including Analytic Hierarchy Process (AHP) and three-phase Quality Function Deployment for Environment (QFDE), weighted objective evaluation method, Morphological Chart, Theory of Inventive Problem Solving (TRIZ) and Blue Ocean Strategy (BOS). Material selection is performed initially by using multi-criteria decision making tools such as AHP and QFDE. Both of these tools suggest the most suitable natural fibre and polymer matrix for the sugar palm fibre-reinforced polymer matrix composites. Prior to this, market investigation is conducted to obtain specific requirements of sugar palmbased products. Application of sugar palm fibre-reinforced polymer matrix composites can be found in the development of boats, tables and automotive anti-roll bars, as the sugar palm fibre is the most suitable natural fibre for these types of applications. In summary, the design process of natural fibre composite-based product should be incorporated with the total design method in order to obtain the optimum design solution. In this study, the design process is limited at the conceptual stage and further study must be conducted, including detailed simulations and experiments.
\end{abstract}

